\title{
The impact of mindfulness-based interventions on symptom burden, positive psychological outcomes, and biomarkers in cancer patients
}

This article was published in the following Dove Press journal:

Cancer Management and Research

I June 2015

Number of times this article has been viewed

\author{
Codie R Rouleau' \\ Sheila N Garland ${ }^{2}$ \\ Linda E Carlson ${ }^{3}$ \\ 'Department of Psychology, Faculty \\ of Arts, University of Calgary, \\ Calgary, AB, Canada; ${ }^{2}$ Department \\ of Family Medicine and Community \\ Health, Perelman School of \\ Medicine, University of Pennsylvania, \\ Philadelphia, PA, USA; ${ }^{3}$ Department \\ of Oncology, Cumming School of \\ Medicine, University of Calgary, \\ Calgary, AB, Canada
}

\begin{abstract}
Research on the use of mindfulness-based stress reduction and related mindfulnessbased interventions (MBIs) in cancer care has proliferated over the past decade. MBIs have aimed to facilitate physical and emotional adjustment to life with cancer through the cultivation and practice of mindfulness (ie, purposeful, nonjudgmental, moment-to-moment awareness). This descriptive review highlights three categories of outcomes that have been evaluated in MBI research with cancer patients - namely, symptom reduction, positive psychological growth, and biological outcomes. We also examine the clinical relevance of each targeted outcome, while describing recently published original studies to highlight novel applications of MBIs tailored to individuals with cancer. Accumulating evidence suggests that participation in a MBI contributes to reductions in psychological distress, sleep disturbance, and fatigue, and promotes personal growth in areas such as quality of life and spirituality. MBIs may also influence markers of immune function, hypothalamic-pituitary-adrenal axis regulation, and autonomic nervous system activity, though it remains unclear whether these biological changes translate to clinically important health benefits. We conclude by discussing methodological limitations of the extant literature, and implications of matching MBIs to the needs and preferences of cancer patients. Overall, the growing popularity of MBIs in cancer care must be balanced against scientific evidence for their impact on specific clinical outcomes.
\end{abstract}

Keywords: mindfulness-based intervention, cancer, mindfulness-based stress reduction, mindfulness-based cancer recovery, outcomes

\section{Introduction}

Cancer is a leading cause of mortality worldwide, with 1.7 million new diagnoses and 585,720 deaths estimated during 2014 in the United States alone. ${ }^{1,2}$ Diagnosis, treatment, and survivorship are associated with a complex array of stressors including treatment side effects; changes in daily activities; disrupted social, family, and occupational roles; and the possibility of cancer recurrence and death. ${ }^{3-5}$ To buffer the effects of these and other stressors, the use of integrative mind-body therapies for cancer care is common. Recent data suggest that $32 \%$ of cancer survivors report current or past use of mind-body interventions such as meditation, yoga, and stress management to promote emotional and physical well-being and to reduce psychological distress. ${ }^{6,7}$

One type of mind-body intervention that has been well studied in cancer populations is mindfulness-based stress reduction (MBSR), a program designed to assist individuals in coping with physical and/or emotional challenges through the cultivation and practice of mindfulness. ${ }^{8}$ The program is semimanualized, meaning that sessions are structured and follow general principles, but they also allow the interventionist 
to flexibly adapt session content based on participant needs and concerns. Mindfulness is a concept rooted in ancient Buddhist tradition that refers to purposeful, nonjudgmental, moment-to-moment awareness. ${ }^{9}$ This is in contrast to the experience of automatically reacting to experience, ruminating about the past, and worrying about the future, which may be prominent in individuals facing a life-threatening diagnosis such as cancer. ${ }^{10}$ The ability to engage in mindful awareness is associated with improved psychological distress and physical health symptoms in populations with chronic illness, including cancer. ${ }^{11,12}$

MBSR was first developed by Kabat-Zinn et al in the late 1970 s to help relieve suffering among individuals with chronic pain, anxiety, and other unexplained medical symptomatology. ${ }^{8,13}$ The traditional MBSR program involves eight weekly 150-minute sessions consisting of didactic instruction, group discussion, formal meditation practice (eg, body scan, sitting meditation, walking meditation, loving kindness), gentle hatha yoga, plus a 6-hour silent meditation retreat. ${ }^{8}$ Essential components also include regular homebased mindfulness meditation and the incorporation of mindfulness into everyday activities. This framework serves as the basis for various MBSR adaptations with various formats and durations, collectively called mindfulness-based interventions (MBIs), that have been used with cancer patients. Two formalized MBIs that have been evaluated in oncology populations include mindfulness-based cognitive therapy (MBCT), ${ }^{21,22}$ which combines the MBSR curriculum with principles of cognitive therapy and was originally intended for the treatment of depression, and mindfulness-based cancer recovery $(\mathrm{MBCR}),{ }^{10}$ one of the most widely evaluated cancer-specific adaptations of MBSR that involves eight 90-minute group classes with content tailored to the unique stressors and experiences associated with cancer. We refer to programs as MBIs if they were developed from Kabat-Zinn's original MBSR framework and if they do not involve only meditation or yoga.

The literature on MBIs for patients with cancer has been synthesized elsewhere several times between 2005 and 2015 in meta-analyses, ${ }^{23-29}$ literature reviews, ${ }^{12,30-34}$ and book chapters. ${ }^{35-37}$ The aim of this paper is to add to this literature by providing a nonsystematic, descriptive review of $\mathrm{MBI}$ outcomes that have been empirically evaluated in cancer populations, with a focus on recently published original studies to highlight research trends and novel applications. The process used to retrieve studies involved an initial search for review papers on MBIs for cancer patients using search terms such as "mindfulness", "mindfulness-based interventions", "mindfulness-based stress reduction", "mindfulness-based cancer recovery", "mindfulness-based cognitive therapy", "cancer", and/or "oncology" in PubMed, PsycINFO, and Google Scholar, followed by a search within reference lists for additional review papers. We then completed an additional search for original studies published since 2014 that had not been included in review articles. Readers are encouraged to consult existing review papers and original articles for more systematic details of methods, samples, and outcomes that have been evaluated in MBI studies.

We outline three categories of outcomes that have been most widely studied - symptom reduction, positive psychological outcomes, and biomarkers - and describe why each outcome might represent a suitable target of intervention in cancer care. Understanding the range of benefits individuals might experience following MBI participation will help ensure interventions are consistent with a particular patient's goals, values, and clinical characteristics, which could impact adherence and outcomes. ${ }^{38-41}$ This review could serve as a springboard for future investigations into the following questions: which desired outcomes can be targeted by MBIs? And for whom might MBIs be well-suited?

\section{Symptom reduction}

Individuals with cancer are prone to multiple symptoms associated with the disease, its treatment, and comorbid conditions. Symptoms may include psychological distress, sleep disturbance, shortness of breath, nausea, diarrhea, pain, lymphedema, vasomotor symptoms, and anemia. ${ }^{38,42} \mathrm{MBIs}$ may play an important role in helping patients manage mental and physical health symptoms, and in reducing the impact of cancer-related symptoms on overall functioning and quality of life. ${ }^{42,43}$ The most widely evaluated symptoms in MBI studies with cancer patients include psychological distress, sleep disturbance, and fatigue, so these are outlined below. It is noteworthy that preliminary evidence exists for the use of MBIs to reduce other cancer symptoms such as pain, sexual difficulties, and hot flashes ${ }^{20,44-46}$ and that MBIs have been widely used to alleviate some of these symptoms (especially pain) in noncancer populations..$^{12,47,48}$

\section{Psychological distress}

Individuals living with cancer commonly experience psychological distress throughout the illness trajectory. ${ }^{49,50}$ Psychological distress is a multidimensional concept that exists along a continuum, and is most commonly assessed using measures of affective states, symptoms of stress, anxiety, and depressed mood. ${ }^{12,51,52}$ It is estimated that $30 \%-43 \%$ of cancer 
patients exceed clinical cutoffs for psychological distress on self-report instruments. ${ }^{52} \mathrm{Up}$ to $38 \%$ meet diagnostic criteria for major depression and $18 \%$ meet criteria for an anxiety disorder, though estimates vary depending on the measurement method and cancer type. ${ }^{49,53}$ Psychological distress can interfere with the ability to cope with cancer and, while still very controversial, some research suggests that persistent high levels of distress may adversely impact cancer progression and survival. ${ }^{51,54,55}$

Psychological distress also represents the most frequently evaluated outcome in clinical studies of MBIs with cancer populations. ${ }^{12,31}$ To our knowledge, six meta-analyses have been published to date that examine the association of MBI participation with measures of psychological distress in people living with cancer. ${ }^{23-26,28,29}$ Most recently, Zainal et al ${ }^{28}$ examined nine studies, ${ }^{15,56-61,85,101}$ including two randomized controlled trials (RCTs), ${ }^{15,61}$ that investigated 6- to 8-week MBIs among women with breast cancer. The results indicate medium to large pooled effects on symptoms of stress, depression, and anxiety. Medium to large effects are also reported in prior meta-analyses of MBIs among cancer patients for improvements in depressed mood and anxiety, ${ }^{23,26,29}$ perceived stress and mood disturbance, ${ }^{29}$ distress, ${ }^{25}$ as well as aggregated mental health outcomes (ie, anxiety, depressed mood, stress, and psychological components of quality of life). ${ }^{24} \mathrm{~A}$ recent $\mathrm{RCT}$ of the traditional MBSR program among 336 women with stage I-III breast cancer indicated that medium to large improvements in depressed mood and anxiety, relative to usual care, were maintained at the 12-month follow-up. ${ }^{62}$ Further, there is growing evidence that MBIs are superior to active control conditions on some measures of distress, including depressed mood, anxiety, and stress symptoms. ${ }^{61,63}$

Narrative reviews and clinical practice guidelines suggest that there is strong evidence for the use of MBIs to improve symptoms of stress, anxiety, and depression among individuals coping with cancer. ${ }^{12,38}$ However, most original studies have utilized uncontrolled, pre-post designs; have primarily recruited early-stage, nonmetastatic breast cancer patients; and have measured affective symptoms using selfreport rather than formal diagnostic procedures. In addition, heightened baseline distress is not consistently used as an inclusion criterion in MBI studies. It therefore remains unclear whether MBIs are appropriate for the treatment of anxiety and/or mood disorders in cancer patients, whether some results would be more pronounced if participants were screened for distress or, in pre-post studies, whether reductions in distress are attributable to the intervention. To date, no MBI studies have selectively recruited cancer patients with diagnosed depression or anxiety; however, there is evidence from pre-post studies in noncancer populations that MBIs are associated with large effects on anxious and depressive symptoms in patients with psychological disorders. ${ }^{64}$ Since 2014, there has been ongoing work to remedy some of these limitations by examining the use of MBIs to reduce distress in oncology samples with diagnoses other than breast cancer, ${ }^{14,17,65-68}$ relatively greater severity of illness, ${ }^{68,69}$ other phases of illness including end of life and survivorship, ${ }^{20,68}$ and with measurement of distress using randomized designs and comparison groups..$^{20,65,66,70}$

\section{Sleep disturbance}

Sleep is a significant problem for many cancer patients. Prevalence estimates suggest that the risk of insomnia symptoms is increased two- to three-fold in cancer patients, and that symptoms persist for years following cancer treatment in up to $44 \%$ of patients. ${ }^{71,72}$ Sleep disturbances such as insomnia can be caused or worsened by treatment side effects, pain, and psychological distress that are common in cancer, and they can be maintained by maladaptive sleep behaviors, dysfunctional cognitions about sleep, and physiological arousal. ${ }^{71,73}$ Despite the common co-occurrence of insomnia with psychological distress, including anxiety and depression, ${ }^{74}$ insomnia symptoms represent a distinct clinical entity that may warrant its own focus in psychosocial interventions. ${ }^{75}$

To capitalize upon potential benefits of mindfulness for alleviating disturbed sleep, a number of RCTs have tested the impact of MBIs on sleep parameters in oncology populations, including four published in 2014 alone. ${ }^{14,16,20,70,76-79}$ Some of these studies report associations between MBIs and improvements in self-reported insomnia severity, ${ }^{14,70}$ sleep quality, ${ }^{20,78}$ sleep onset latency and wake time after sleep onset, ${ }^{70}$ sleep difficulties/disturbance, ${ }^{16,79}$ and in actigraphic measures of sleep quality/quantity, ${ }^{70,77}$ with some changes observed 12 weeks following program completion. ${ }^{77}$ Associations between MBIs and improvements in sleep have not always been significant relative to active treatment and waitlist comparison groups, ${ }^{70,76,77,79}$ and some findings may not be significant after correction for potential type I error. ${ }^{16,77}$ It is possible that more robust results would be observed if patients were selected for pre-existing sleep difficulty, given that most trials have not involved the a priori decision to target sleep, but rather included sleep-related measures as ancillary outcomes of larger RCTs. ${ }^{14,16,20,76-78}$

A notable exception is the recent study by Garland et al, ${ }^{70}$ in which all patients were required to meet research 
and diagnostic criteria for insomnia. This study reported the results from a RCT comparing MBCR to cognitive-behavioral therapy for insomnia among 111 patients with various cancer diagnoses. This trial was unique in that patients chose to participate in order to address insomnia rather than to learn meditation skills; they were also blinded to the treatment options at study consent, and they were informed only about their own assigned intervention. A noninferiority analysis indicated post-treatment insomnia symptoms were less severe in participants who completed cognitive-behavioral therapy relative to those who completed MBCR; however, MBCR was noninferior to cognitive-behavioral therapy for improving insomnia severity at the 3-month follow-up, with both groups showing subthreshold insomnia symptoms. These findings suggest that while MBCR may be slower to take effect on disturbed sleep relative to cognitive-behavioral therapy, both treatments produce clinically significant improvements in sleep in the longer term.

In general, it appears that MBIs may represent a treatment option for cancer patients with sleep difficulties, and that the effect of MBIs on sleep can be significant in light of the chronicity of insomnia in many individuals (eg, a mean of 7 years insomnia duration in Garland et $\mathrm{al}^{70}$ ). Although there are currently no evidence-based guidelines on the use of MBIs for improving sleep in cancer patients, presumably due to insufficient evidence to date, it is encouraging to see renewed interest in the use of MBIs for cancer-related sleep difficulties since the first sleep outcomes of a 6-week MBSR program in cancer patients were published in $2003 .{ }^{76}$ Additional, welldesigned, adequately powered controlled trials are needed to further establish the effect of MBIs in patients with insomnia who seek out this form of treatment.

\section{Fatigue}

Another frequently investigated cancer symptom in MBI research has been cancer-related fatigue (CRF), a persistent pattern of tiredness and diminished energy that is inconsistent with changes in sleep or activity levels and interferes with day-to-day functioning. ${ }^{80,81} \mathrm{CRF}$ is one of the most common and distressing symptoms of cancer, with prevalence estimates suggesting that $59 \%-100 \%$ of cancer patients experience fatigue, and that 9\%-56\% experience clinically significant $\mathrm{CRF}^{42,82,83}$ For up to $40 \%$ of patients, problems with fatigue persist for months or years following cancer treatment. $^{84}$

Among studies that have evaluated fatigue as an outcome of MBIs in cancer populations, ${ }^{20,58,69,85-88}$ two RCTs that have specifically targeted fatigue as a primary outcome deserve mention. ${ }^{14,89}$ Johns et al $^{14}$ examined an abbreviated MBI for reducing fatigue among 35 post-treatment cancer survivors with CRF. The intervention consisted of seven 2-hour classes that involved education about CRF, training and practice in mindfulness meditation, and gentle hatha yoga, as well as 20 minutes of daily home practice. Relative to a waitlist group, patients who completed the MBI reported lower fatigue severity, lower fatigue interference, and greater vitality postprogram and at 1-month follow-up. The results corresponded to large effects and persisted after correction for multiple comparisons and statistical adjustment for baseline fatigue, mindfulness skills, and participation in recent mental health treatment. It is also noteworthy that all participants were retained from baseline through to 1-month follow-up, intervention attendance was high (88\%), and the interventionist was blind to patient outcomes. Similarly, van der Lee and Garssen ${ }^{89}$ randomized 100 post-treatment cancer patients with significant fatigue to either a waitlist control or to an eight-session program, modified from the original MBCT program described earlier, ${ }^{22}$ to include psychoeducation about CRF in the place of depression content and an added exercise on coping with interpersonal boundaries. Following adjustment for baseline fatigue, fatigue severity was lower in MBCT participants relative to controls, which corresponded to a large effect that was maintained at the 6-month follow-up.

These findings support the possibility of tailoring MBIs to alleviate $\mathrm{CRF}$, and they are consistent with the conclusions of review articles suggesting that MBIs are likely to be effective in the treatment of $\mathrm{CRF}^{81}$ and that there is moderate evidence for the use of MBIs to alleviate CRF. ${ }^{90}$ However, MBIs for fatigue also need to be investigated among patients with more severe cancer, given that a recent nonrandomized feasibility study involving a small group of 19 women with metastatic breast cancer indicated that standard 8-week MBSR was not associated with reductions in CRF from baseline to 4 months postprogram. ${ }^{69}$ Future work is also needed to confirm the utility of fatigue-specific MBIs with larger samples and to examine potential biobehavioral mechanisms through which mindfulness might influence CRF.

\section{Positive psychological outcomes}

There is growing recognition that the prominent focus on pathological outcomes in psychosocial oncology overlooks potential experiences of personal growth and meaningmaking that can arise from the cancer experience, ${ }^{43,91}$ which may serve as an important buffer to psychological distress. ${ }^{43}$ Similarly, it has been suggested that psychosocial 
interventions in cancer populations should target and evaluate not just symptom reduction, but also positive psychological outcomes that may arise..$^{13,43,91}$

The most frequently evaluated positive psychosocial outcome in MBI research has been quality of life - that is, “individuals' perception of their position in life in the context of the culture and value systems in which they live and in relation to their goals, expectations, standards, and concerns". ${ }^{92}$ Musial et $\mathrm{al}^{25}$ reported on four single-arm studies and two RCTs representing 248 cancer patients with mixed diagnoses who had received some form of MBI. ${ }^{16,58,86,93-95}$ Overall, there was a small effect of these MBIs on postprogram quality of life. Improvements in self-reported general well-being were also observed 1 year following an 8-week MBI that included cognitive therapy elements in 47 patients with mixed cancer diagnoses. ${ }^{86}$ Consistent with these findings, clinical practice guidelines indicate that there is strong evidence that meditation practice, including formal MBI participation, is associated with improved quality of life in patients treated for breast cancer. ${ }^{38}$

In addition to improvements in quality of life, several single-group studies and RCTs with cancer patients have demonstrated that MBIs are associated with positive changes in other areas such as spirituality, positive affect, meaningfulness, acceptance, comfort, happiness, relaxation, and posttraumatic growth..$^{20,46,61,96}$ However, positive psychological outcomes of MBIs have not consistently been superior to usual care or active comparison groups. ${ }^{15,96}$ Pooled results from two RCTs with oncology populations indicate no significant group differences between MBIs and usual care in terms of spiritual well-being outcomes (ie, the quality of life dimension that involves a sense of purpose, peace of mind, and finding comfort in one's spiritual beliefs). ${ }^{15,23,61,97}$ In one of these two studies, though, breast cancer patients in 8-week standard MBSR did show significantly greater improvements in spirituality when compared to both usual care and a nutrition education condition, which were maintained relative to the active control at the 12-month follow-up. ${ }^{61}$

As an example of a recent study that has evaluated positive psychological outcomes from MBIs, Thornton et $\mathrm{al}^{17}$ evaluated a 20 -session intervention that incorporated MBSR with sessions focused on values-based goal setting, stress management, social support, assertive communication, sexuality, and health behaviors. Thirty-two women with recurrent breast or gynecological cancer received the intervention over a 7-month period. There were medium to large improvements from baseline to postprogram in terms of hope, mindfulness, positive affect, and quality of life. Interestingly, greater mindfulness and hope were related to lower mood disturbance, anxiety, and depressive symptoms, suggesting that positive psychological outcomes tend to cooccur with reductions in psychological distress.

Qualitative studies also help generate a subjective understanding of positive psychological benefits that cancer patients might experience from mindfulness training. Responses in interviews, focus groups, and open-ended questionnaires with oncology samples who completed mindfulness training (either on its own or as part of a structured MBI) reveal themes of spirituality, personal growth, optimism, peacefulness, confidence, acceptance, openness, connectedness, happiness, general satisfaction, and appreciation of the group process and shared experience. ${ }^{68,69,98-102}$ For example, Visser et al ${ }^{99}$ interviewed nine women with advanced breast cancer about their experiences during a group-based MBI that involved eight weekly 2.5-hour sessions and a 1-day silent retreat. Themes revealed in the qualitative interviews suggest that patients felt that mindfulness training led them to have greater awareness of their thoughts and feelings, increased acceptance of their health condition, improved energy, better communication with significant others, and social support from other participants. ${ }^{99}$ Similarly, van den Hurk et $\mathrm{al}^{68}$ interviewed six patients with lung cancer and five partners of lung cancer patients who had participated in a MBSR program that included psychoeducation about grief. Participants reported that mindfulness practice had initiated a positive process of change, characterized by experiences such as standing still (eg, relaxing, taking time for themselves), being aware, insight into thoughts and feelings, letting go, changing behavior, and acceptance. Existing quantitative and qualitative findings suggest that patients may achieve a number of positive psychological benefits as a result of MBI participation, although future research is needed to clarify inconsistent findings.

\section{Biomarkers}

In addition to lessening some of the symptoms associated with cancer and promoting personal growth, evidence is beginning to emerge that MBI participation is associated with alterations in potentially important biological markers of stress and health. ${ }^{12,55,103}$ Biomarkers that have been shown to change following participation by cancer patients in MBIs include measures of immune function, ${ }^{20,94,95,104,105}$ hypothalamicpituitary-adrenal axis regulation, ${ }^{63,94,104-107}$ prostate-specific antigen levels, ${ }^{108}$ and autonomic nervous system activity; $46,94,109$ not all biological changes, however, were observed relative to control groups. Literature reviews have generally concluded that MBIs show small but significant effects on biomarkers and physical health. ${ }^{24,29}$ Matchim et $\mathrm{al}^{29}$ reviewed four studies of MBIs in cancer patients and reported a small significant 
effect on biological outcomes in most studies, ${ }^{94,95,105,106}$ with the exception of a large effect of MBCR on cytokine production at 6- and 12-month follow-up in an uncontrolled study with breast and prostate cancer patients. ${ }^{94}$ Similarly, a review of eight studies reported that MBIs show a small effect on physical health parameters including biomarkers (eg, levels of cortisol and interleukin 6, natural killer cell activity) and physical symptoms (eg, self-reported gastrointestinal and neurologic issues) in cancer populations. ${ }^{24}$

Since the publication of these literature reviews, two RCTs have investigated the impact of $\mathrm{MBSR}^{110}$ and $\mathrm{MBCR}^{111}$ on additional biomarkers - namely, telomere length and telomerase activity - in breast cancer patients. Telomeres are specialized structures that protect chromosome ends, with shorter telomere length associated with cellular aging, whereas telomerase is a ribonucleoprotein enzyme involved in telomere maintenance and elongation. ${ }^{12}$ Both studies report promising results, such that telomere length was maintained following MBCR relative to a minimal intervention control condition, ${ }^{111}$ and telomerase activity improved more in MBSR relative to usual care and was sustained at 12-week follow-up. ${ }^{110}$ These two studies add to the growing literature on the potential effects of MBIs on biomarkers of health, given that shorter telomere length and telomerase activity have been shown to predict cancer mortality. ${ }^{113-115}$

Taken together, these findings suggest that various biological parameters may serve as subtle but significant markers of treatment response to MBIs, and they may represent mechanisms through which psychosocial function could impact physical health. ${ }^{55,116}$ However, it will be crucial in future research to ensure that immune-endocrine outcomes are straightforward to interpret and have relevance for health. ${ }^{117}$ For instance, whether enhanced natural killer cell function is desirable or not may depend on the type and stage of cancer, the phase of treatment, and the baseline level of this biomarker of immune system activity. Further, biomarker response to MBIs may differ as a function of patient sex, cancer type, and baseline biologic values. ${ }^{106,107,109}$ The causal pathways linking MBIs to both stress reduction and biological activity are also unclear, such that biological changes can be a cause and/or effect of behavioral comorbidities such as psychological distress, depressed mood, fatigue, and impaired sleep. ${ }^{103}$ Therefore, the relationship between biomarker response to MBIs and long-term health or cancer symptoms is not well understood.

\section{Discussion and implications}

MBIs have become increasingly popular programs to assist cancer patients as they navigate the experience of living with the disease. It is clear that the mindfulness approach is a versatile one, with an abundance of benefits that may arise from realizing that one can "slow down and see things as they are, without blinders, and learn ways to hold the strong emotions and sensations that arise". ${ }^{12}$ In returning to the initial question of which desired outcomes can be targeted by MBIs, the most consistently demonstrated benefit in cancer and noncancer populations is reduced psychological distress (perhaps, in part, because it is also the most routinely assessed outcome). ${ }^{12,118}$ Additional benefits may include reductions in sleep disturbance and fatigue, as well as improvements in overall quality of life, positive well-being, and spirituality. There is also growing evidence that MBIs may influence biological parameters, though it remains to be seen whether or not the changes observed translate into clinically significant health benefits for the patient.

A commonly reported reason for the use of MBIs and other complementary therapies by cancer patients is to promote general self-care and wellness, though some patients also desire specific outcomes such as enhanced immune function and reduced pain, distress, and insomnia. ${ }^{6,119}$ This suggests the importance of using existing empirical data on patient outcomes to ensure MBIs are matched to patient needs and preferences. For example, an individual seeking improvements in distress, sleep, fatigue, and/or positive psychological well-being may be satisfied with the program given the demonstrated ability of MBIs to influence these outcomes. In contrast, it is less clear whether MBIs can influence other symptoms a patient might hope to alleviate, such as pain, sexual issues, nausea, or cognitive dysfunction. ${ }^{38}$ It is also possible that MBI retention could be improved if relevant content is added to interventions that target specific symptoms. Among patients joining a MBI to treat insomnia, for example, it may be important to provide education about insomnia and evidence supporting the use of mindfulness practice for disturbed sleep. Additional research is needed to assess whether the addition of symptom-specific modules can favorably influence MBI uptake, adherence, and outcomes.

Though some patients may join MBIs with the hope of improving biological outcomes, ${ }^{6}$ it is currently premature to conclude that these interventions can influence variables such as immune function or cellular aging in clinically meaningful ways. Research on biomarker outcomes has shown small effects, inconsistent findings, and has yet to demonstrate links to health outcomes such as cancer progression and longevity. Therefore, it is important to ensure prospective MBI participants have realistic expectations about the biological outcomes they might achieve, while still appreciating that research on biomarkers may contribute to 
our understanding about mechanisms of action and treatment response. ${ }^{107,110,120,121}$ It will be interesting for future studies to continue examining how biomarkers relate to changes in mindfulness and psychosocial outcomes, including symptom reduction and positive well-being.

In terms of general research limitations, high attrition from MBIs, lack of long-term follow-up, and non-RCT designs continue to be methodological issues in some studies. ${ }^{24,33}$ The majority of research on MBIs also involves relatively small samples of self-selecting, educated, middle-aged White women with breast cancer, which could limit generalizability to individuals with different symptoms, prognoses (eg, those in the palliative stage of cancer), sociodemographics (eg, males), and views about mindfulness. ${ }^{27,28,33}$ Although participation in MBIs may assist cancer patients in dealing with symptoms of distress, sleep disturbance, or fatigue, it is currently not clear whether these interventions are useful to those living with other symptoms. As well, since these types of interventions only appeal to a subset of patients, the utility of the broad application of MBIs to all cancer patients and survivors is unknown. In addition, variability across studies in terms of outcome measures, cancer stage, and intervention content makes it difficult to compare findings or evaluate the "active ingredients" of MBIs. ${ }^{25}$ Some authors have indicated a need for greater investigation of how MBIs compare to other efficacious interventions (eg, cognitive-behavioral therapy), and how various "doses" of MBI (eg, program duration, amount of daily mindfulness practice) compare in their ability to improve outcomes. ${ }^{12,34,70,122}$

Recent and ongoing research shows promise in some of these areas. For example, Kenne Sarenmalm et al ${ }^{123}$ plan to perform a 5-year follow-up in a RCT of MBSR for psychological distress in breast cancer patients, which will provide relatively long-term data on MBI outcomes. Also, van den Hurk et $\mathrm{al}^{68}$ published a mixed methods pilot study on MBSR for lung cancer patients (53\% men) and their partners, demonstrating the feasibility of delivering MBIs within a nonbreast cancer sample. There has also been methodological progress with respect to abbreviated and online versions of MBIs designed to reduce the burden of program participation, ${ }^{14,48,65,123}$ and the inclusion of partners or close significant others in MBIs. ${ }^{68,104,124,125}$ For example, Wood et $\mathrm{al}^{125}$ recently published a session-by-session protocol for adapting MBCT to the caregivers of cancer patients. Partner involvement could be of added value considering that partners commonly report psychological distress that is inter-related with patient distress, and that there is preliminary evidence for the idea that partner participation in MBIs can reduce caregiver burden. ${ }^{68,126,127}$
Overall, the growing popularity of MBSR and other MBIs for cancer patients must be balanced against scientific evidence for their impact on specific clinical outcomes. ${ }^{122}$ With the potential appeal of mindfulness training as a panacea to promote all facets of health and well-being, clinicians must be aware of the current state of evidence for the use of MBIs to facilitate physical and emotional adjustment to cancer.

\section{Acknowledgments}

Dr Linda E Carlson holds the Enbridge Research Chair in Psychosocial Oncology at the University of Calgary Cumming School of Medicine, cofunded by the Canadian Cancer Society Alberta/Northwest Territories Division and Alberta Cancer Foundation. Dr Sheila N Garland is a postdoctoral fellow funded by a Canadian Institutes for Health Research (CIHR) Bisby Fellowship. Codie R Rouleau is funded by a CIHR Doctoral Award and an Alberta Innovates Health Solutions (AIHS) Graduate Studentship.

\section{Disclosure}

The authors report no conflicts of interest in this work.

\section{References}

1. Stewart BW, Wild CP, editors. World Cancer Report 2014. Lyon, France: International Agency for Research on Cancer; 2014.

2. American Cancer Society. Cancer Facts and Figures 2014. Atlanta, GA: American Cancer Society; 2014. Available from: http://www. cancer.org/acs/groups/content/@research/documents/webcontent/ acspc-042151.pdf. Accessed May 3, 2015.

3. Fallowfield L, Jenkins V. Psychosocial/survivorship issues in breast cancer: are we doing better? J Natl Cancer Inst. 2015;107(1):335.

4. Hewitt M, Breen N, Devesa S. Cancer prevalence and survivorship issues: analyses of the 1992 National Health Interview Survey. J Natl Cancer Inst. 1999;91(17):1480-1486.

5. Alfano CM, Rowland JH. Recovery issues in cancer survivorship: a new challenge for supportive care. Cancer J. 2006;12(5):432-443.

6. Mao JJ, Palmer CS, Healy KE, Desai K, Amsterdam J. Complementary and alternative medicine use among cancer survivors: a populationbased study. J Cancer Surviv. 2011;5(1):8-17.

7. Deng G, Cassileth B. Complementary or alternative medicine in cancer care-myths and realities. Nat Rev Clin Oncol. 2013;10(11): 656-664.

8. Kabat-Zinn J. Full Catastrophe Living: Using the Wisdom of Your Body and Mind to Face Stress, Pain, and Illness. New York, NY: Delacourt; 1990.

9. Bishop SR, Lau M, Shapiro S, et al. Mindfulness: a proposed operational definition. Clinical Psychology: Science and Practice. 2004;11:230-241.

10. Carlson LE, Speca M. Mindfulness-Based Cancer Recovery. Oakland, CA: New Harbinger; 2010.

11. Dobkin PL, Zhao Q. Increased mindfulness - the active component of the mindfulness-based stress reduction program? Complement Ther Clin Pract. 2011;17(1):22-27.

12. Carlson LE. Mindfulness-based interventions for physical conditions: a narrative review evaluating levels of evidence. ISRN Psychiatry. 2012;2012:651583.

13. Shapiro SL, Carlson LE. The Art and Science of Mindfulness: Integrating Mindfulness into Psychology and the Helping Professions. Washington, DC: American Psychological Association Publications; 2009. 
14. Johns SA, Brown LF, Beck-Coon K, Monahan PO, Tong Y, Kroenke K. Randomized controlled pilot study of mindfulness-based stress reduction for persistently fatigued cancer survivors. Psychooncology. Epub August 17, 2014.

15. Lengacher CA, Johnson-Mallard V, Post-White J, et al. Randomized controlled trial of mindfulness-based stress reduction (MBSR) for survivors of breast cancer. Psychooncology. 2009;18(12):1261-1272.

16. Monti DA, Peterson C, Kunkel EJ, et al. A randomized, controlled trial of mindfulness-based art therapy (MBAT) for women with cancer. Psychooncology. 2006;15(5):363-373.

17. Thornton LM, Cheavens JS, Heitzmann CA, Dorfman CS, Wu SM, Andersen BL. Test of mindfulness and hope components in a psychological intervention for women with cancer recurrence. J Consult Clin Psychol. 2014;82(6):1087-1100.

18. Henderson VP, Massion AO, Clemow L, Hurley TG, Druker S, Hébert JR. A randomized controlled trial of mindfulness-based stress reduction for women with early-stage breast cancer receiving radiotherapy. Integr Cancer Ther. 2013;12(5):404-413.

19. Bartley T. Mindfulness-Based Cognitive Therapy for Cancer. West Sussex, UK: Wiley-Blackwell; 2012.

20. Bower JE, Crosswell AD, Stanton AL, et al. Mindfulness meditation for younger breast cancer survivors: A randomized controlled trial. Cancer. 2015;121(8):1231-1240.

21. Segal ZV, Williams JM, Teasdale JD. Mindfulness-Based Cognitive Therapy for Depression. New York, NY: The Guilford Press; 2002.

22. Segal Z, Williams M, Teasdale J. Mindfulness-Based Cognitive Therapy for Depression: A New Approach to Preventing Relapse. 1st ed. New York, NY: The Guilford Press; 2001.

23. Cramer H, Lauche R, Paul A, Dobos G. Mindfulness-based stress reduction for breast cancer-a systematic review and meta-analysis. Curr Oncol. 2012;19(5):e343-e352.

24. Ledesma D, Kumano H. Mindfulness-based stress reduction and cancer: a meta-analysis. Psychooncology. 2009;18(6):571-579.

25. Musial F, Büssing A, Heusser P, Choi KE, Ostermann T. Mindfulnessbased stress reduction for integrative cancer care: a summary of evidence. Forsch Komplementmed. 2011;18(4):192-202.

26. Piet J, Würtzen H, Zachariae R. The effect of mindfulness-based therapy on symptoms of anxiety and depression in adult cancer patients and survivors: a systematic review and meta-analysis. J Consult Clin Psychol. 2012;80(6):1007-1020.

27. Shneerson C, Taskila T, Gale N, Greenfield S, Chen YF. The effect of complementary and alternative medicine on the quality of life of cancer survivors: a systematic review and meta-analyses. Complement Ther Med. 2013;21(4):417-429.

28. Zainal NZ, Booth S, Huppert FA. The efficacy of mindfulness-based stress reduction on mental health of breast cancer patients: a metaanalysis. Psychooncology. 2013;22(7):1457-1465.

29. Matchim Y, Armer JM, Stewart BR. Mindfulness-based stress reduction among breast cancer survivors: a literature review and discussion. Oncol Nurs Forum. 2011;38(2):E61-E71.

30. Mackenzie MJ, Carlson LE, Speca M. Mindfulness-based stress reduction (MBSR) in oncology: rationale and review. Evidence-Based Integrative Medicine. 2005;2(3):139-145.

31. Matchim Y, Armer JM. Measuring the psychological impact of mindfulness meditation on health among patients with cancer: a literature review. Oncol Nurs Forum. 2007;34(5):1059-1066.

32. Ott MJ, Norris RL, Bauer-Wu SM. Mindfulness meditation for oncology patients: a discussion and critical review. Integr Cancer Ther. 2006;5(2): 98-108.

33. Shennan C, Payne S, Fenlon D. What is the evidence for the use of mindfulness-based interventions in cancer care? A review. Psychooncology. 2011;20(7):681-697.

34. Smith JE, Richardson J, Hoffman C, Pilkington K. Mindfulness-based stress reduction as supportive therapy in cancer care: systematic review. J Adv Nurs. 2005;52(3):315-327.

35. Carlson LE. Meditation and physical health. In: West MA, editor. The Psychology of Meditation: Research and Practice. 2nd ed. Oxford, UK: Oxford University Press. In Press.
36. Carlson LE. Mindfulness meditation and yoga for cancer patients. In: Holland JC, Breitbart WS, Jacobsen PB, Loscalzo MJ, McCorkle R, Butow PN, editors. Psycho-Oncology. 3rd ed. Oxford, UK: Oxford University Press; 2015:492.

37. Shapiro S, Carlson L. Mindfulness-based interventions for physical health. In: The Art and Science of Mindfulness: Integrating Mindfulness into Psychology and the Helping Professions. Washington, DC: American Psychological Association Publications; 2009:75-91.

38. Greenlee H, Balneaves LG, Carlson LE, et al; Society for Integrative Oncology Guidelines Working Group. Clinical practice guidelines on the use of integrative therapies as supportive care in patients treated for breast cancer. J Natl Cancer Inst Monogr. 2014;2014(50): 346-358.

39. Kemper K, Bulla S, Krueger D, Ott MJ, McCool JA, Gardiner P. Nurses' experiences, expectations, and preferences for mind-body practices to reduce stress. BMC Complement Altern Med. 2011;11:26.

40. Würtzen H, Dalton SO, Andersen KK, et al. Who participates in a randomized trial of mindfulness-based stress reduction (MBSR) after breast cancer? A study of factors associated with enrollment among Danish breast cancer patients. Psychooncology. 2013;22(5): 1180-1185.

41. Carlson LE, Tamagawa R, Stephen J, et al. Tailoring mind-body therapies to individual needs: patients' program preference and psychological traits as moderators of the effects of mindfulness-based cancer recovery and supportive-expressive therapy in distressed breast cancer survivors. J Natl Cancer Inst Monogr. 2014;2014(50):308-314.

42. Esther Kim JE, Dodd MJ, Aouizerat BE, Jahan T, Miaskowski C. A review of the prevalence and impact of multiple symptoms in oncology patients. J Pain Symptom Manage. 2009;37(4):715-736.

43. Shapiro SL, Lopez AM, Schwartz GE, et al. Quality of life and breast cancer: relationship to psychosocial variables. J Clin Psychol. 2001;57(4):501-519.

44. Brotto LA, Heiman JR, Goff B, et al. A psychoeducational intervention for sexual dysfunction in women with gynecologic cancer. Arch Sex Behav. 2008;37(2):317-329.

45. Brotto LA, Heiman JR. Mindfulness in sex therapy: applications for women with sexual difficulties following gynecologic cancer. Sex Relation Ther. 2007;22(1):3-11.

46. Bauer-Wu S, Sullivan AM, Rosenbaum E, et al. Facing the challenges of hematopoietic stem cell transplantation with mindfulness meditation: a pilot study. Integr Cancer Ther. 2008;7(2):62-69.

47. Chiesa A, Serretti A. Mindfulness-based interventions for chronic pain: a systematic review of the evidence. J Altern Complement Med. 2011;17(1):83-93.

48. Dowd H, Hogan MJ, McGuire BE, et al. Comparison of an online mindfulness-based cognitive therapy intervention with online pain management psychoeducation: a randomized controlled study. Clin J Pain. Epub January 6, 2015.

49. Massie MJ. Prevalence of depression in patients with cancer. $J$ Natl Cancer Inst Monogr. 2004;(32):57-71.

50. Zabora JR, Blanchard CG, Smith ED, et al. Prevalence of psychological distress among cancer patients across the disease continuum. Journal of Psychosocial Oncology. 1997;15(2):73-87.

51. National Comprehensive Cancer Network, Inc. Practice Guidelines in Oncology - v.1.2002: Distress Management. Version 1. Jentintown, PA: National Comprehensive Cancer Network, Inc.; 2002.

52. Zabora J, BrintzenhofeSzoc K, Curbow B, Hooker C, Piantadosi S. The prevalence of psychological distress by cancer site. Psychooncology. 2001;10(1):19-28.

53. Stark D, Kiely M, Smith A, Velikova G, House A, Selby P. Anxiety disorders in cancer patients: their nature, associations, and relation to quality of life. J Clin Oncol. 2002;20(14):3137-3148.

54. Brown KW, Levy AR, Rosberger Z, Edgar L. Psychological distress and cancer survival: a follow-up 10 years after diagnosis. Psychosom Med. 2003;65(4):636-643.

55. Lutgendorf SK, Sood AK. Biobehavioral factors and cancer progression: physiological pathways and mechanisms. Psychosom Med. 2011;73(9):724-730. 
56. Matousek RH, Pruessner JC, Dobkin PL. Changes in the cortisol awakening response (CAR) following participation in mindfulness-based stress reduction in women who completed treatment for breast cancer. Complement Ther Clin Pract. 2011;17(2):65-70.

57. Matousek RH, Dobkin PL. Weathering storms: a cohort study of how participation in a mindfulness-based stress reduction program benefits women after breast cancer treatment. Curr Oncol. 2010;17(4): $62-70$.

58. Lengacher CA, Johnson-Mallard V, Barta M, et al. Feasibility of a mindfulness-based stress reduction program for early-stage breast cancer survivors. J Holist Nurs. 2011;29(2):107-117.

59. Tacón AM, Caldera YM, Ronaghan C. Mindfulness-based stress reduction in women with breast cancer. Families, Systems, and Health 2004;22(2):193-203

60. Tacón AM. Mindfulness effects on symptoms of distress in women with cancer. Journal of Cancer Pain and Symptom Palliation. 2006;2(2): $17-22$.

61. Henderson VP, Clemow L, Massion AO, Hurley TG, Druker S, Hébert JR. The effects of mindfulness-based stress reduction on psychosocial outcomes and quality of life in early-stage breast cancer patients: a randomized trial. Breast Cancer Res Treat. 2012;131(1):99-109.

62. Würtzen H, Dalton SO, Elsass P, et al. Mindfulness significantly reduces self-reported levels of anxiety and depression: results of a randomised controlled trial among 336 Danish women treated for stage I-III breast cancer. Eur J Cancer. 2013;49(6):1365-1373.

63. Carlson LE, Doll R, Stephen J, et al. Randomized controlled trial of mindfulness-based cancer recovery versus supportive expressive group therapy for distressed survivors of breast cancer. J Clin Oncol. 2013 31(25):3119-3126.

64. Hofmann SG, Sawyer AT, Witt AA, Oh D. The effect of mindfulnessbased therapy on anxiety and depression: A meta-analytic review. J Consult Clin Psychol. 2010;78(2):169-183.

65. Zernicke KA, Campbell TS, Speca M, McCabe-Ruff K, Flowers S, Carlson LE. A randomized wait-list controlled trial of feasibility and efficacy of an online mindfulness-based cancer recovery program: the eTherapy for cancer applying mindfulness trial. Psychosom Med. 2014;76(4):257-267.

66. Stafford L, Thomas N, Foley E, et al. Comparison of the acceptability and benefits of two mindfulness-based interventions in women with breast or gynecologic cancer: a pilot study. Support Care Cancer. 2015;23(4):1063-1071.

67. Schellekens MP, van den Hurk DG, Prins JB, et al. Study protocol of a randomized controlled trial comparing mindfulness-based stress reduction with treatment as usual in reducing psychological distress in patients with lung cancer and their partners: the MILON study. BMC Cancer. 2014;14:3.

68. van den Hurk DG, Schellekens MP, Molema J, Speckens AE, van der Drift MA. Mindfulness-based stress reduction for lung cancer patients and their partners: results of a mixed methods pilot study. Palliat Med. Epub February 20, 2015

69. Eyles C, Leydon GM, Hoffman CJ, et al. Mindfulness for the self-management of fatigue, anxiety, and depression in women with metastatic breast cancer: a mixed methods feasibility study. Integr Cancer Ther. 2015;14(1):42-56

70. Garland SN, Carlson LE, Stephens AJ, Antle MC, Samuels C, Campbell TS. Mindfulness-based stress reduction compared with cognitive behavioral therapy for the treatment of insomnia comorbid with cancer: a randomized, partially blinded, noninferiority trial. J Clin Oncol. 2014;32(5):449-457.

71. Savard J, Savard MH. Insomnia and cancer: prevalence, nature, and nonpharmacologic treatment. Sleep Med Clin. 2013;8(3):373-387.

72. Savard J, Morin CM. Insomnia in the context of cancer: a review of a neglected problem. J Clin Oncol. 2001;19(3):895-908.

73. Harvey AG. A cognitive model of insomnia. Behav Res Ther. 2002; 40(8):869-893.

74. American Psychiatric Association. Diagnostic and Statistical Manual of Mental Disorders. 5th ed. Arlington, VA: American Psychiatric Publishing; 2013.
75. Buysse DJ, Angst J, Gamma A, Ajdacic V, Eich D, Rössler W. Prevalence, course, and comorbidity of insomnia and depression in young adults. Sleep. 2008;31(4):473-480.

76. Shapiro SL, Bootzin RR, Figueredo AJ, Lopez AM, Schwartz GE. The efficacy of mindfulness-based stress reduction in the treatment of sleep disturbance in women with breast cancer: an exploratory study. J Psychosom Res. 2003;54(1):85-91.

77. Lengacher CA, Reich RR, Paterson CL, et al. The effects of mindfulness-based stress reduction on objective and subjective sleep parameters in women with breast cancer: a randomized controlled trial. Psychooncology. 2015;24(4):424-432.

78. Andersen SR, Würtzen H, Steding-Jessen M, et al. Effect of mindfulnessbased stress reduction on sleep quality: results of a randomized trial among Danish breast cancer patients. Acta Oncol. 2013;52(2) 336-344.

79. Lipschitz DL, Kuhn R, Kinney AY, Donaldson GW, Nakamura Y. Reduction in salivary $\alpha$-amylase levels following a mind-body intervention in cancer survivors - an exploratory study. Psychoneuroendocrinology. 2013;38(9):1521-1531.

80. Portenoy RK, Itri LM. Cancer-related fatigue: guidelines for evaluation and management. Oncologist. 1999;4(1):1-10.

81. Mitchell SA, Hoffman AJ, Clark JC, et al. Putting evidence into practice: an update of evidence-based interventions for cancer-related fatigue during and following treatment. Clin J Oncol Nurs. 2014;18 Suppl: 38-58.

82. Donovan KA, McGinty HL, Jacobsen PB. A systematic review of research using the diagnostic criteria for cancer-related fatigue. Psychooncology. 2013;22(4):737-744.

83. Weis J. Cancer-related fatigue: prevalence, assessment and treatment strategies. Expert Rev Pharmacoecon Outcomes Res. 2011;11(4): 441-446.

84. Servaes P, Verhagen S, Bleijenberg G. Determinants of chronic fatigue in disease-free breast cancer patients: a cross-sectional study. Ann Oncol. 2002;13(4):589-598.

85. Matchim Y, Armer JM, Stewart BR. Effects of mindfulness-based stress reduction (MBSR) on health among breast cancer survivors. West $J$ Nurs Res. 2011;33(8):996-1016.

86. Kieviet-Stijnen A, Visser A, Garssen B, Hudig W. Mindfulness-based stress reduction training for oncology patients: patients' appraisal and changes in well-being. Patient Educ Couns. 2008;72(3): 436-442.

87. Garland SN, Tamagawa R, Todd SC, Speca M, Carlson LE. Increased mindfulness is related to improved stress and mood following participation in a mindfulness-based stress reduction program in individuals with cancer. Integr Cancer Ther. 2013;12(1):31-40.

88. Carlson LE, Garland SN. Impact of mindfulness-based stress reduction (MBSR) on sleep, mood, stress and fatigue symptoms in cancer outpatients. Int J Behav Med. 2005;12(4):278-285.

89. van der Lee ML, Garssen B. Mindfulness-based cognitive therapy reduces chronic cancer-related fatigue: a treatment study. Psychooncology. 2012;21(3):264-272.

90. Pachman DR, Price KA, Carey EC. Nonpharmacologic approach to fatigue in patients with cancer. Cancer J. 2014;20(5):313-318.

91. Casellas-Grau A, Font A, Vives J. Positive psychology interventions in breast cancer. A systematic review. Psychooncology. 2014;23(1): 9-19.

92. World Health Organization. Programme on Mental Health: WHOQOL Measuring Quality of Life. Geneva, Switzerland: World Health Organization; 1997.

93. Foley E, Baillie A, Huxter M, Price M, Sinclair E. Mindfulness-based cognitive therapy for individuals whose lives have been affected by cancer: a randomized controlled trial. J Consult Clin Psychol. 2010; 78(1):72-79.

94. Carlson LE, Speca M, Faris P, Patel KD. One year pre-post intervention follow-up of psychological, immune, endocrine and blood pressure outcomes of mindfulness-based stress reduction (MBSR) in breast and prostate cancer outpatients. Brain Behav Immun. 2007;21(8): $1038-1049$. 
95. Carlson LE, Speca M, Patel KD, Goodey E. Mindfulness-based stress reduction in relation to quality of life, mood, symptoms of stress, and immune parameters in breast and prostate cancer outpatients. Psychosom Med. 2003;65(4):571-581.

96. Garland SN, Carlson LE, Cook S, Lansdell L, Speca M. A nonrandomized comparison of mindfulness-based stress reduction and healing arts programs for facilitating post-traumatic growth and spirituality in cancer outpatients. Support Care Cancer. 2007;15(8): 949-961.

97. Bredle JM, Salsman JM, Debb SM, Arnold BJ, Cella D. Spiritual wellbeing as a component of health-related quality of life: the Functional Assessment of Chronic Illness Therapy - Spiritual Well-Being scale. Religions. 2011;2(1):77-94.

98. Chadwick P, Newell T, Skinner C. Mindfulness groups in palliative care: a pilot qualitative study. Spirituality and Health International. 2008;9(3):135-144.

99. Visser A, de Witte T, Speckens AE, Prins JB, van Laarhoven HW. A qualitative analysis of experiences of patients with metastatic breast cancer participating in a mindfulness-based intervention. Palliat Med. 2015;29(2):182-183.

100. Mackenzie MJ, Carlson LE, Munoz M, Speca M. A qualitative study of self-perceived effects of mindfulness-based stress reduction (MBSR) in a psychosocial oncology setting. Stress Health. 2007;23(1):59-69.

101. Dobkin PL. Mindfulness-based stress reduction: what processes are at work? Complement Ther Clin Pract. 2008;14(1):8-16.

102. Hoffman CJ, Ersser SJ, Hopkinson JB. Mindfulness-based stress reduction in breast cancer: a qualitative analysis. Complement Ther Clin Pract. 2012;18(4):221-226.

103. Miller AH, Ancoli-Israel S, Bower JE, Capuron L, Irwin MR. Neuroendocrine-immune mechanisms of behavioral comorbidities in patients with cancer. J Clin Oncol. 2008;26(6):971-982.

104. Lengacher CA, Kip KE, Barta M, et al. A pilot study evaluating the effect of mindfulness-based stress reduction on psychological status, physical status, salivary cortisol, and interleukin-6 among advancedstage cancer patients and their caregivers. J Holist Nurs. 2012;30(3): $170-185$.

105. Witek-Janusek L, Albuquerque K, Chroniak KR, Chroniak C, Durazo-Arvizu R, Mathews HL. Effect of mindfulness based stress reduction on immune function, quality of life and coping in women newly diagnosed with early stage breast cancer. Brain Behav Immun. 2008;22(6):969-981.

106. Carlson LE, Speca M, Patel KD, Goodey E. Mindfulness-based stress reduction in relation to quality of life, mood, symptoms of stress and levels of cortisol, dehydroepiandrosterone sulfate (DHEAS) and melatonin in breast and prostate cancer outpatients. Psychoneuroendocrinology. 2004;29(4):448-474.

107. Bränström R, Kvillemo P, Akerstedt T. Effects of mindfulness training on levels of cortisol in cancer patients. Psychosomatics. 2013;54(2): 158-164.

108. Saxe GA, Hébert JR, Carmody JF, et al. Can diet in conjunction with stress reduction affect the rate of increase in prostate specific antigen after biochemical recurrence of prostate cancer? J Urol. 2001;166(6): 2202-2207.

109. Campbell TS, Labelle LE, Bacon SL, Faris P, Carlson LE. Impact of Mindfulness-Based Stress Reduction (MBSR) on attention, rumination and resting blood pressure in women with cancer: a waitlist-controlled study. J Behav Med. 2012;35(3):262-271.

110. Lengacher CA, Reich RR, Kip KE, et al. Influence of mindfulnessbased stress reduction (MBSR) on telomerase activity in women with breast cancer (BC). Biol Res Nurs. 2014;16(4):438-447.
111. Carlson LE, Beattie TL, Giese-Davis J, et al. Mindfulness-based cancer recovery and supportive-expressive therapy maintain telomere length relative to controls in distressed breast cancer survivors. Cancer. 2015;121(3):476-484.

112. Greider CW. Telomere length regulation. Annu Rev Biochem. 1996;65:337-365.

113. Rossi D, Lobetti Bodoni C, Genuardi E, et al. Telomere length is an independent predictor of survival, treatment requirement and Richter's syndrome transformation in chronic lymphocytic leukemia. Leukemia. 2009;23(6):1062-1072.

114. Ghaffari SH, Shayan-Asl N, Jamialahmadi AH, Alimoghaddam K, Ghavamzadeh A. Telomerase activity and telomere length in patients with acute promyelocytic leukemia: indicative of proliferative activity, disease progression, and overall survival. Ann Oncol. 2008;19(11): 1927-1934.

115. Willeit $\mathrm{P}$, Willeit J, Mayr A, et al. Telomere length and risk of incident cancer and cancer mortality. JAMA. 2010;304(1):69-75.

116. Matousek RH, Dobkin PL, Pruessner J. Cortisol as a marker for improvement in mindfulness-based stress reduction. Complement Ther Clin Pract. 2010;16(1):13-19.

117. Miller GE, Cohen S. Psychological interventions and the immune system: a meta-analytic review and critique. Health Psychol. 2001;20(1):47-63.

118. Khoury B, Lecomte T, Fortin G, et al. Mindfulness-based therapy: a comprehensive meta-analysis. Clin Psychol Rev. 2013;33(6): 763-771.

119. Weitz MV, Fisher K, Lachman VD. The journey of women with breast cancer who engage in mindfulness-based stress reduction: a qualitative exploration. Holist Nurs Pract. 2012;26(1):22-29.

120. Labelle LE, Campbell TS, Faris P, Carlson LE. Mediators of mindfulness-based stress reduction (MBSR): assessing the timing and sequence of change in cancer patients. $J$ Clin Psychol. 2015;71(1):21-40

121. Ong JC, Ulmer CS, Manber R. Improving sleep with mindfulness and acceptance: a metacognitive model of insomnia. Behav Res Ther. 2012;50(11):651-660.

122. Bishop SR. What do we really know about mindfulness-based stress reduction? Psychosom Med. 2002;64(1):71-83.

123. Kenne Sarenmalm E, Mårtensson LB, Holmberg SB, Andersson BA, Odén A, Bergh I. Mindfulness based stress reduction study design of a longitudinal randomized controlled complementary intervention in women with breast cancer. BMC Complement Altern Med. 2013; $13: 248$.

124. Birnie K, Garland SN, Carlson LE. Psychological benefits for cancer patients and their partners participating in mindfulness-based stress reduction (MBSR). Psychooncology. 2010;19(9):1004-1009.

125. Wood AW, Gonzalez J, Barden SM. Mindful caring: using mindfulness-based cognitive therapy with caregivers of cancer survivors. J Psychosoc Oncol. 2015;33(1):66-84.

126. Moser MT, Künzler A, Nussbeck F, Bargetzi M, Znoj HJ. Higher emotional distress in female partners of cancer patients: prevalence and patient-partner interdependencies in a 3-year cohort. Psychooncology. 2013;22(12):2693-2701.

127. Hodges LJ, Humphris GM, Macfarlane G. A meta-analytic investigation of the relationship between the psychological distress of cancer patients and their carers. Soc Sci Med. 2005;60(1):1-12. 


\section{Publish your work in this journal}

Cancer Management and Research is an international, peer-reviewed open access journal focusing on cancer research and the optimal use of preventative and integrated treatment interventions to achieve improved outcomes, enhanced survival and quality of life for the cancer patient The journal welcomes original research, clinical \& epidemiological studies, reviews \& evaluations, guidelines, expert opinion \& commentary, case reports \& extended reports. The manuscript management system is completely online and includes a very quick and fair peerreview system, which is all easy to use. Visit http://www.dovepress.com/ testimonials.php to read real quotes from published authors.

Submit your manuscript here: http://www.dovepress.com/cancer-management-and-research-journal 